\title{
Leptin signaling in intestinal epithelium mediates resistance to enteric infection by Entamoeba histolytica
}

\author{
X Guo ${ }^{1,6}$, MR Roberts ${ }^{1}$, SM Becker ${ }^{1}$, B Podd ${ }^{1}$, Y Zhang ${ }^{2}$, SC Chua Jr ${ }^{3}$, MG Myers Jr $^{4}$, P Duggal ${ }^{5}$, \\ ER Houpt ${ }^{1}$ and WA Petri Jr ${ }^{1}$
}

Leptin is an adipocytokine that links nutrition to immunity. Previous observation that a genetic polymorphism in the leptin receptor affected susceptibility to Entamoeba histolytica infection led to the hypothesis that leptin signaling has a protective role during intestinal amebic infection. In this study we show that mice lacking the functional leptin receptor developed devastating mucosal destruction after $E$. histolytica infection. Bone marrow chimera experiments demonstrated that leptin receptor expressed on hematopoietic cells was not sufficient to confer resistance. Similarly, peripheral knockout of the leptin receptor rendered animals susceptible, indicating that central expression of the leptin receptor was not sufficient to confer protection. The site of leptin action was localized to the gut via an intestinal epithelium-specific deletion of the leptin receptor, which rendered mice susceptible to infection and mucosal destruction by the parasite. Mutation of tyrosine 985 or 1138 in the intracellular domain of the leptin receptor, which mediates signaling through the SH2-containing tyrosine phosphatase/extracellular signal-regulated kinase (SHP2/ ERK) and signal transducer and activator of transcription 3 (STAT3) pathways, respectively, demonstrated that both were important for mucosal protection. We conclude that leptin-mediated resistance to amebiasis is via its actions on intestinal epithelium rather than hematopoietic cells or the brain, and requires leptin receptor signaling through both the STAT3 and SHP2/ERK pathways.

\section{INTRODUCTION}

Malnutrition represents a significant health problem in the developing world. Increasing evidence has supported an epidemiological association between susceptibility to infection and malnutrition. ${ }^{1-5}$ Since 1999 , we have been conducting a prospective case-control study to examine the genetic and environmental factors that can increase the risk of diarrheal diseases in Dhaka, Bangladesh. We found that malnourished individuals were more likely to acquire Entamoeba histolytica-associated diarrheal illness. ${ }^{4,5}$ Given that malnourished children had lower leptin levels than the well-nourished ones, ${ }^{6}$ we asked whether the increased susceptibility to amebiasis in malnourished individuals could be partly explained by the diminished function of leptin, an adipocyte-derived hormone that acts as a link between nutrition and immunity. ${ }^{3,7,8}$ Supporting a link between leptin and amebiasis, we also found that susceptibility to amebic infection in the children was associated with an amino acid substitution (Q223R) in the extracellular domain of the leptin receptor (P. Duggal et al., unpublished data).

Leptin has a pleiotropic role in mammalian physiology. Leptin acts centrally to regulate food intake and energy expenditure through its functional long form receptor (OB-Rb; $\mathrm{LRb}$ ) in the hypothalamus. ${ }^{9,10}$ In the periphery, leptin promotes the survival and function of a variety of immune cells ${ }^{11-19}$ and acts as a proinflammatory cytokine that orchestrates the inflammatory response toward a Th1 phenotype while suppressing Th2 and regulatory T-cell functions..$^{20,21}$ This is of particular interest in the setting of E. histolytica infection, as a Th1 response

${ }^{1}$ Division of Infectious Diseases and International Health, Department of Medicine, University of Virginia, Charlottesville, Virginia, USA. ${ }^{2}$ Division of Molecular Genetics and Naomi Berrie Diabetes Center, Department of Pediatrics, Columbia University Medical Center, New York, New York, USA. ${ }^{3}$ Departments of Medicine and Neuroscience, Albert Einstein College of Medicine, Bronx, New York, USA. ${ }^{4}$ Departments of Internal Medicine and Molecular and Integrative Physiology, University of Michigan, Ann Arbor, Michigan, USA. ${ }^{5}$ Department of Epidemiology, Johns Hopkins Bloomberg School of Public Health, Baltimore, Maryland, USA. ${ }^{2}$ Present address: Vaccine Research Center, NIAID, National Institutes of Health, Bethesda, Maryland, USA. Correspondence: WA Petri Jr (wap3g@virginia.edu) or ER Houpt (erh6k@virginia.edu) 
(specifically, interferon- $\gamma$ ) is a critical component of protective immunity against amebiasis. ${ }^{22-24}$ An additional potential protective role of leptin against amebiasis is in mucosal immunity, as the leptin receptor is also expressed in the intestinal mucosa. ${ }^{25}$ Indeed, leptin helps maintain intestinal mucosal integrity, ${ }^{26}$ stimulates mucin secretion upon mucosal damage, ${ }^{27}$ and modulates intestinal barrier function via its proproliferative and antiapoptotic effects. ${ }^{28,29}$

The leptin receptor belongs to the type I cytokine receptor family. ${ }^{30}$ Leptin binding to LRb results in the activation and tyrosine phosphorylation of Janus kinase 2 (JAK2), which induces subsequent phosphorylation of conserved tyrosine residues in the intracellular tail of LRb. Each phosphorylated tyrosine residue mediates distinct signals as shown in Figure 1. Phosphorylated Tyr1138 recruits signal transducer and activator of transcription 3 (STAT3) that upon phosphorylation mediates the transcription of suppressor of cytokine signaling (SOCS3) as well as positive effectors of leptin action. Phosphorylated Tyr985 recruits the SH2-containing tyrosine phosphatase (SHP2) and mediates activation of mitogen-activated protein kinase/extracellular signal-regulated kinase

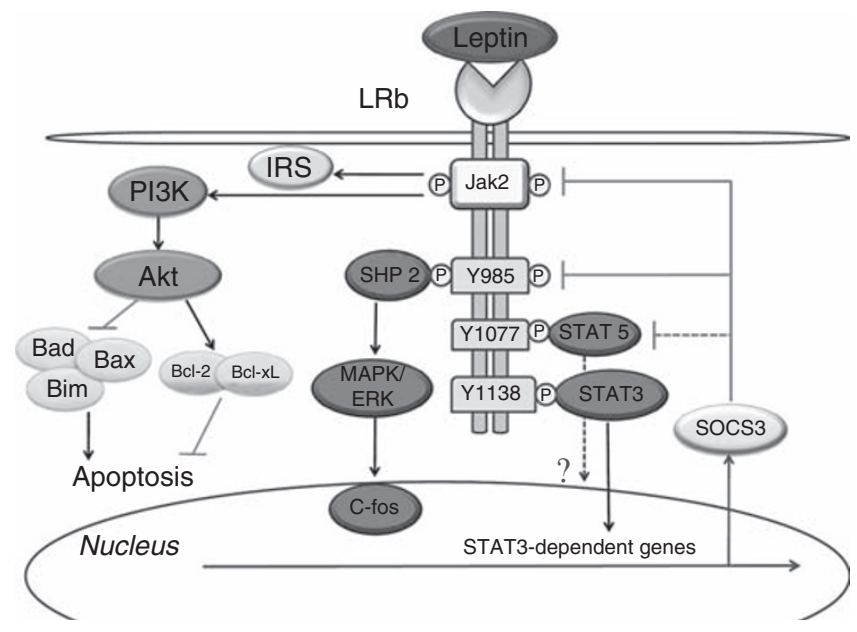

Figure 1 Long form leptin receptor (LRb) signaling. Leptin binding to the long form $L R b$ results in the activation and tyrosine phosphorylation of Janus kinase 2 (JAK2), which induces subsequent phosphorylation of downstream tyrosine residues (Tyr985, Tyr1077, and Tyr1138) in the intracellular tail of LRb. Phosphorylated Tyr1138 binds and mediates the phosphorylation-dependent activation of signal transducer and activator of transcription 3 (STAT3), which activates transcription of suppressor of cytokine signaling 3 (SOCS3) and other positive effectors of leptin action. Phosphorylated Tyr985 recruits the SH2-containing tyrosine phosphatase SHP2, which activates the signaling pathway that culminates in extracellular signal-regulated kinase (ERK) activation. During prolonged stimulation, phosphorylated Tyr985 binds SOCS3 to mediate feedback inhibition of LRb signaling. Phosphorylated Tyr1077 recruits STAT5 to induce STAT5-dependent transcription activation; however, its functional effect is less clear. Signals mediated via phosphorylated Jak2 include insulin receptor substrate protein (IRS) and the phosphoinositide 3-kinase (PI3K)/Akt pathway. The $\mathrm{PI3K} /$ Akt signaling is important for the LRb-mediated antiapoptotic and proproliferative effects via downregulating proapoptotic (e.g., Bad, Bim, Bax, and p53) while upregulating antiapoptotic/survival factors (e.g., B-cell lymphoma 2 (Bcl-2), Bcl-XL, and cyclin D). (Figure adapted from Febbraio, M.A. J. Clin. Invest. 117, 841-849 (2007)).
(MAPK/ERK); during prolonged stimulation, phosphorylated Tyr985 also binds SOCS3 and mediates feedback inhibition of LRb signaling. ${ }^{31-33}$ In addition to the signal pathways coupled to tyrosine residues, some signals emanate directly from Jak2, including the insulin receptor substrate protein, phosphoinositide 3-kinase (PI3K)/Akt pathway, and a minor component of the ERK pathway. ${ }^{31,32}$ It has been reported that PI3K/Akt or SHP2/MAPK/ERK signaling contributes to LRb-mediated antiapoptotic and proproliferative effects. ${ }^{17,28,34}$ LRb Tyr1138/STAT3 and Tyr985/SHP2 signaling in the hypothalamus is crucial for the regulation of neuroendocrine functions such as food intake, energy balance, and reproduction. ${ }^{31-33}$ How these pathways impact the actions of leptin in the periphery and in mucosal immunity remains under investigation.

In this study, we examined the role of leptin in host resistance to E. histolytica infection, and sought to understand the mechanisms by which this adipocytokine confers mucosal protection. Using a series of genetically engineered mice, including those with site-specific disruption/deletion of leptin receptor, we investigated the site of leptin action and the signaling pathways that are important for leptin-mediated protection.

\section{RESULTS \\ Leptin signaling is important in mucosal resistance to intestinal amebiasis}

To test the role of leptin signaling in host resistance to amebiasis, mice genetically deficient in leptin (B6.V-Lep $p^{o b} / j$, ob/ob) and in the functional leptin receptor (B6.Cg-m Lepr $r^{d b} / j$, $\mathrm{db} / \mathrm{db}$ ) were tested for susceptibility to intestinal amebiasis. Compared with the highly resistant C57BL/6 wild-type mice, $\mathrm{ob} / \mathrm{ob}$ and $\mathrm{db} / \mathrm{db}$ mice were significantly more susceptible to the establishment of E. histolytica infection at $48 \mathrm{~h}$, as determined by both cecal amebic antigen and cultural positivity (presented as infection rate; Figure 2a). Interestingly, the $\mathrm{db} / \mathrm{db}$ mice displayed an unusual rapid death phenotype $16 \mathrm{~h}$ after E. histolytica infection: up to $50 \%$ of the $\mathrm{db} / \mathrm{db}$ mice were found dead or euthanized before the scheduled termination time $(48 \mathrm{~h})$ because of a moribund state. The mortality rate of $\mathrm{db} / \mathrm{db}$ mice was significantly higher than that of the wild-type mice $(P<0.01$ at $48 \mathrm{~h})$, and approaching significance compared with ob/ob mice that exhibited intermediate level of mortality $(P=0.07$; Figure $2 \mathbf{b})$. In contrast, the early mortality phenotype was not seen in $\mathrm{db} / \mathrm{db}$ mice sham challenged with conditioned ameba culture media (Figure 2b). The pathology of the infected ceca reflected the more severe course of amebiasis in both ob/ob and db/db mice: whereas infected wild-type mice exhibited normal mucosa hyperplasia (Figure 2c, left), the ceca from ob/ob and $\mathrm{db} / \mathrm{db}$ mice revealed near-complete loss of the epithelium, and substantial tissue disruption associated with invading trophozoites (Figure 2c, middle and right). The sham-infected $\mathrm{db} / \mathrm{db}$ mice displayed normal-looking cecal histology (data not shown). We concluded that the lack of leptin or functional leptin receptor increased susceptibility to intestinal amebiasis in the first $48 \mathrm{~h}$ after infection. 

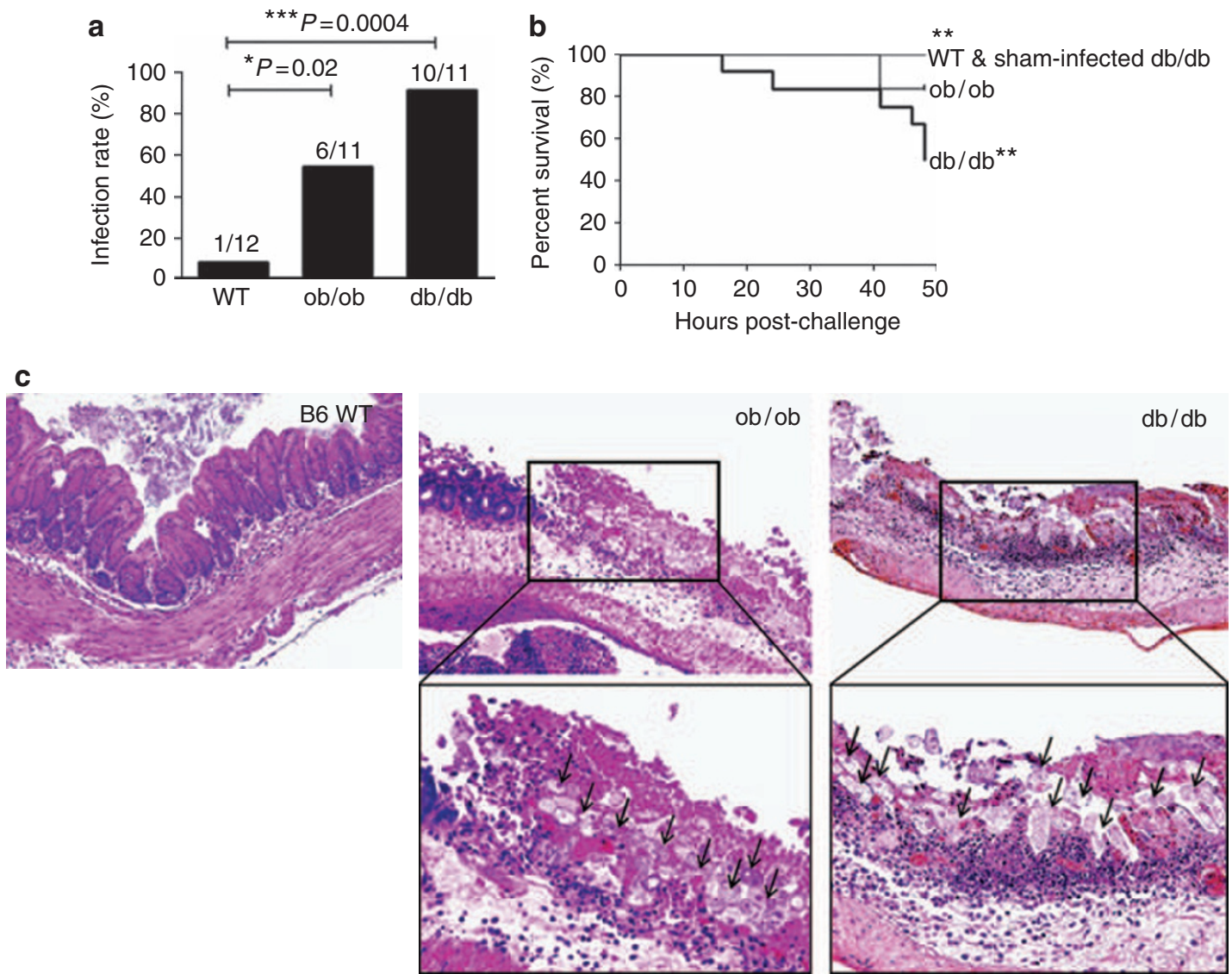

Figure 2 Deficiency in leptin signaling renders the mice more susceptible to intestinal infection by Entamoeba histolytica. (a) Mice genetically deficient in leptin (ob/ob) and in functional leptin receptor $(\mathrm{db} / \mathrm{db})$ were more susceptible to intestinal infection with E. histolytica as determined by cecal amebic antigen and cultural positivity (presented as infection rate). (b) An unusual moribund phenotype was observed in db/db and ob/ob mice that started within $24 \mathrm{~h}$ post-challenge. The Kaplan-Meier survival curves depict time to death for mice challenged with $E$. histolytica in hours. The experiment ended after 48h. Log-rank test for B6 vs. db/db: $P=0.005$ (no B6 wild-type (WT) or sham-challenged db/db mice died, $50 \%$ of infected $\mathrm{db} / \mathrm{db}$ mice died); $\mathrm{db} / \mathrm{db}$ vs. ob/ob: $P=0.09$ (17\% of ob/ob mice died). For each group, $n=12$. (c) Mucosal hyperplasia was observed in the WT mice (B6) that were infected (left), whereas ob/ob (middle) and db/db (right) mice had extensive epithelial loss and trophozoite invasion (arrows). Original magnification: images are $\times 100$, except insets (bottom row) that are $\times 200$.

\section{Identification of the target site on which leptin acts to protect}

Leptin exerts its pleiotropic effects via leptin receptors expressed on many cell types. Hence, the next step toward understanding the role of leptin in mucosal immunity was to identify the relative importance of leptin receptors expressed on intestinal epithelial cells, immune cells, and the central nervous system. To first address whether immune cells are a major target for leptin action, we generated reciprocal bone marrow chimeras between wild-type C57BL/6 (B6) and db/db mice. Specifically, the hematopoietic systems of recipient $\mathrm{B} 6$ or $\mathrm{db} / \mathrm{db}$ mice were ablated by irradiation before transplant of bone marrow from $\mathrm{db} / \mathrm{db}$ or $\mathrm{B} 6$ donors, respectively. Efficient reconstitution of $\mathrm{db} / \mathrm{db} \rightarrow \mathrm{B} 6$ and $\mathrm{B} 6 \rightarrow \mathrm{db} / \mathrm{db}$ chimeras was confirmed by flow cytometric analysis of peripheral blood mononuclear cells stained with Thy 1.1 and 1.2 (Figure 3a). At 9 weeks post-transfer, mice were challenged with $E$. histolytica trophozoites. At $48 \mathrm{~h}$ post-challenge, $\mathrm{B} 6 \rightarrow \mathrm{db} / \mathrm{db}$ chimeras recapitulated the susceptible phenotype of $d b / d b \rightarrow d b / d b$ mice with high amebic antigen (Figure $3 \mathbf{b}$ ), high culture-positivity rate (Figure $3 \mathrm{c}$ ), and high mortality (Figure 3d). Similarly, db/db $\rightarrow$ B6 chimeras exhibited the resistance as seen in B6 $\rightarrow$ B6 group (Figure $\mathbf{3 b}$-d), suggesting that leptin's action on bone marrow-derived immune cells is not sufficient to mediate mucosal resistance to E. histolytica.

To delineate the contribution of central vs. peripheral leptin signaling to mucosal immunity, we generated mice in which leptin receptor signaling was selectively disrupted in peripheral tissues. Specifically, we crossed mice whose LRb exon 17 is flanked by two loxP sequences (Lepr ${ }^{\text {flox/flox }}$ mice), with transgenic mice expressing a tamoxifen (Tam)-inducible Cre recombinase (ROSA26 $6^{\mathrm{Cre}-\mathrm{ERT} 2 /+}$ mice), to produce Lepr ${ }^{\text {flox/flox }}$ ROSA26Cre-ERT2/+ (Cre) mice and Lepr ${ }^{\text {flox/flox }}$ (Non-cre) mice. As exon 17 encodes the Jak2 binding site that is indispensable for downstream LRb signal transduction, deletion of exon 17 results in a disrupted/dysfunctional LRb. ${ }^{35}$ Because Tam has a poor permeability across the blood-brain barrier, Tam treatment of Cre but not control Non-cre mice selectively disrupts leptin receptor signaling in peripheral tissues. A previous report demonstrated that the Tam-induced deletion process was complete shortly after a 5-day Tam treatment, and that the pattern and extent of deletion remained essentially unchanged after that. ${ }^{36}$ The extent of Tam-induced deletion of exon 17 in the brain and cecum was examined at the time when 


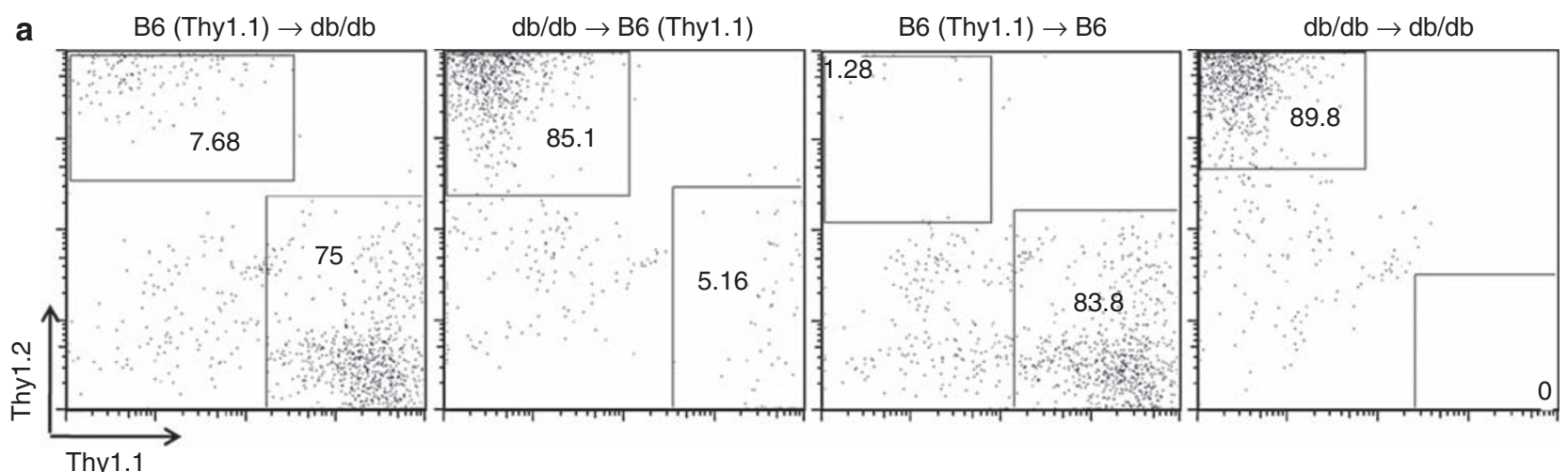

Thy 1.1
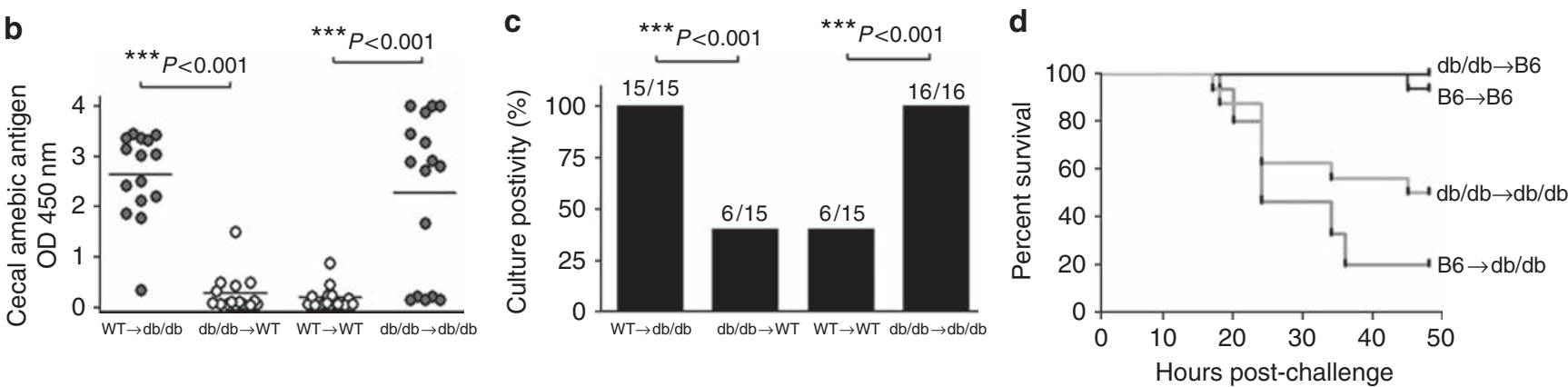

Figure 3 Leptin-mediated resistance to Entamoeba histolytica was not restored by transplant of a wild-type (WT) marrow into db/db mice. Recipient mice were reconstituted intravenous (IV) with bone marrow cells prepared from donor femur and tibia. (a) Engraftment of donor hematopoietic cells were confirmed by fluorescence-activated cell sorting (FACS) for Thy 1.1 and Thy 1.2 at 8 weeks post-transfer on peripheral blood mononuclear cells (PBMCs) ( $n=8$ per group, one representative mouse per group shown). At 9 weeks post-transfer, mice were challenged intracecally with $E$. histolytica trophozoites and killed after $48 \mathrm{~h}$ for evaluation of infection according to (b) cecal amebic antigen, (c) culture positivity, and (d) early mortality. Log-rank test for difference in survival: $\mathrm{db} / \mathrm{db}$ recipients vs. WT recipients: $P<0.0001 ; \mathrm{db} / \mathrm{db}$ to $\mathrm{db} / \mathrm{db}$ vs. WT to $\mathrm{db} / \mathrm{db}: P=0.11 ; N=16$ per group.

E. histolytica-infected mice were killed (day 4 post infection, 22 days after Tam treatment) by PCR and gel electrophoresis, using primers flanking the two loxP sites (Figure 4a). Extensive deletion of exon 17 was detected in the cecum of Tam-Cre mice (Figure 4a, "Tam-Cre," "Cec" lanes). No deletion was detected in the brain of Tam-Cre mice (Figure 4a, "Tam-Cre," "Br" lanes), and no deletion occurred in either brain or cecum of untreated Cre or Tam-treated Non-cre mice (Figure 4a, "Cre" and "Tam-Non-cre"). To test the susceptibility of TamCre mice to intestinal amebiasis, Tam-treated Cre and Non-cre mice were challenged with E. histolytica. Increased susceptibility was seen in the Tam-treated Cre mice compared with the Non-cre controls as determined by culture positivity and amebic antigen (Figure 4b). Furthermore, disease severity was also increased in Tam-Cre mice as measured by substantial mucosal destruction and inflammation (Figure 4c, top) whereas the E. histolytica-infected controls mainly exhibited submucosal edema or modest disruption of mucosal architecture with mild infiltration (Figure 4c, bottom). No histological difference was seen between the uninfected Cre and Non-cre animals before or after Tam treatment (data not shown). These data indicate that leptin receptor signaling in the brain is not sufficient to provide protection against intestinal amebiasis.

To determine whether intestinal epithelium was a direct target of leptin for the mucosal resistance to amebiasis, mice selectively lacking functional leptin receptors in intestinal epithelial cells were generated. Specifically, we crossed mice harboring floxed leptin receptor alleles (Lepr flox/flox $)$ with mice expressing Cre under the control of the villin promoter (Vil-Cre), ${ }^{37}$ generating Lepr ${ }^{\text {flox/flox }}$ Vil-Cre (Vil-Cre KO) and Lepr ${ }^{\text {flox/flox }}$ (flox/flox control) mice. PCR was performed with genomic DNA from the cecum, liver, or the intestine-associated adipose tissue to confirm the tissue specificity of Cre recombination. The deleted allele was detectable in the cecum of Vil-Cre KO but not flox/flox mice. Furthermore, no deletion was detected in non-intestinal tissues of the Vil-Cre KO mice (Figure 5a). Deletion of leptin receptor was further confirmed by cecal immunohistochemistry: the flox/flox control mice exhibited intense staining for leptin receptor at basolateral and apical membranes of intestinal epithelial cells (Figure 5b, top and middle), whereas the Vil-Cre KO cecum only revealed background staining at the intestinal lining (Figure 5b, bottom). Vil-Cre KO mice and the Non-cre flox/flox were then challenged with E. histolytica and killed 4 days later. As shown in Figure 5c, mice homozygously deleted for lepr in the intestine exhibited a statistically higher infection rate than the flox/flox controls as determined by cecal amebic antigen and cultural positivity ( 7 out of 12 vs. 1 out of $9, P<0.05$ ). An extensive mucosal erosion and epithelial loss were observed in the cecum of infected Vil-Cre KO mice, in contrast with the relatively normal histology of the control cecum (Figure 5d). These results indicated that leptin receptor expressed on the intestinal epithelium was a critical target site for leptinmediated protection against amebiasis. 
a

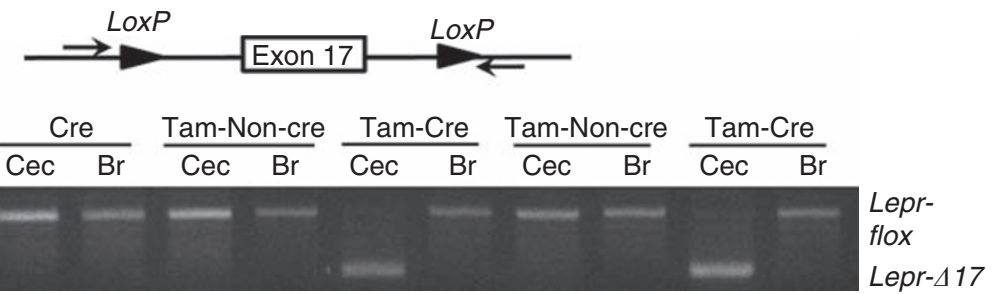

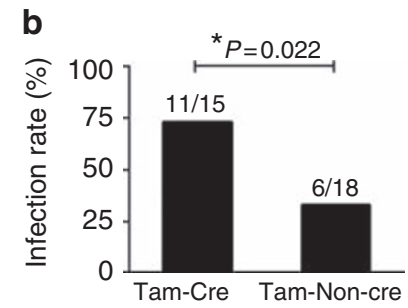
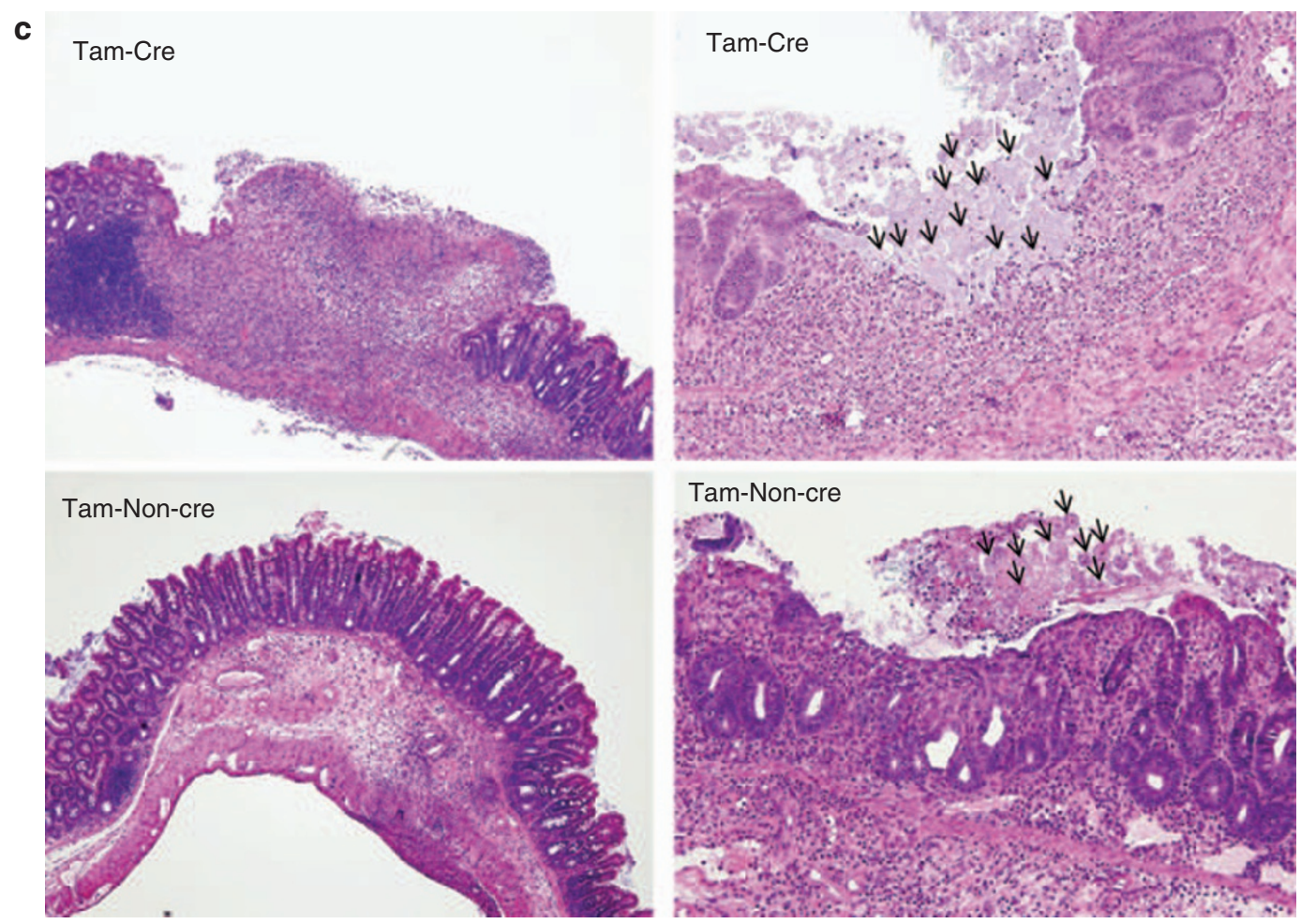

Figure 4 Peripheral disruption of leptin receptor resulted in increased susceptibility to Entamoeba histolytica. Lepr flox/flox/Cre (+) and Lepr flox/flox/Cre $(-)$ mice were treated with tamoxifen (Tam) for 5 days. (a) After Tam treatment, genomic DNA was extracted from the brain (Br) and the cecum (Cec), and PCR was performed using primers recognizing the flanking sequences of the LoxP to examine the deletion of leptin receptor exon 17. (b) Tam-Cre mice with a peripheral disruption of the leptin receptor and control mice (Tam-Non-cre) with intact leptin receptor were intracecally infected with E. histolytica, and susceptibility to amebiasis was assessed by cecal amebic antigen and cultural positivity (presented as infection rate). (c) Representative histopathology of infected ceca from Tam-Cre (top) mice showed more severe destruction of mucosal architecture with large ulcerations and extensive infiltration compared with the infected Tam-Non-cre controls (bottom). Amebic trophozoites are indicated by arrows. Original magnifications: left $\times 40$, right $\times 100$.

\section{Identification of the key leptin receptor signaling pathways for leptin-mediated protection}

To examine the contributions of downstream LRb signaling pathways to leptin's protective effect in the setting of E. histolytica infection, the relative susceptibility of mice engineered to express mutations in key tyrosine residues (Tyr1138 and Tyr985) in the LRb intracellular tail was evaluated. Mice carrying a serine replacement of Tyr1138 in the leptin receptor (S1138) have disrupted STAT3 signaling, and mice carrying a leucine substitution at Tyr985 (L985) have disrupted SHP2/MAPK/ERK signaling and SOCS3-mediated feedback inhibition. Both tyrosine substitutions rendered mice more susceptible to intestinal amebiasis according to the cecal antigen and cultural positivity, although none of the variants presented the early mortality phenotype seen for the $\mathrm{db} / \mathrm{db}$ mouse (Figure 6a and $\mathbf{b}$ ). Interestingly, the histopathology differed in these two variants upon infection: the majority of the infected S1138 mice showed a disrupted intestinal mucosa with ulcerative/necrotic lesions in the cecum (Figure 6c). In contrast, infected L985 mice mainly presented with mucosal hyperplasia and little mucosal destruction (Figure 6d). Invading trophozoites could be found in some infected S1138 mice along the lamina propria and basement membrane (Figure 6e, arrows). The naive S1138 and L985 mice both exhibited normal intestinal morphology without detectable differences (data not shown). These data indicate that both the STAT3 and SHP2/MAPK/ERK signaling pathways contribute to leptin's protective effects.

\section{DISCUSSION}

Our results show for the first time that the adipocytokine leptin has a protective role in mucosal resistance to amebic infection, and does so primarily via leptin receptors expressed on intestinal epithelial cells. Furthermore, both the Y1138/STAT3 and Y985/ SHP2/ERK signal transduction pathways of LRb are required for leptin-mediated protection. 
a

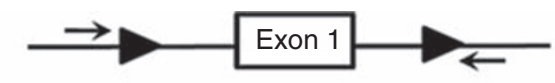

flox/flox

Vil-Cre lepr KO

\begin{tabular}{llll}
\hline Liv & Cec Liv & Cec & Liv Cec Liv Cec Adi Cec Liv Cec Liv Cec \\
\hline
\end{tabular}

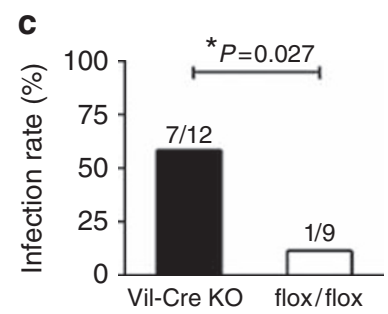

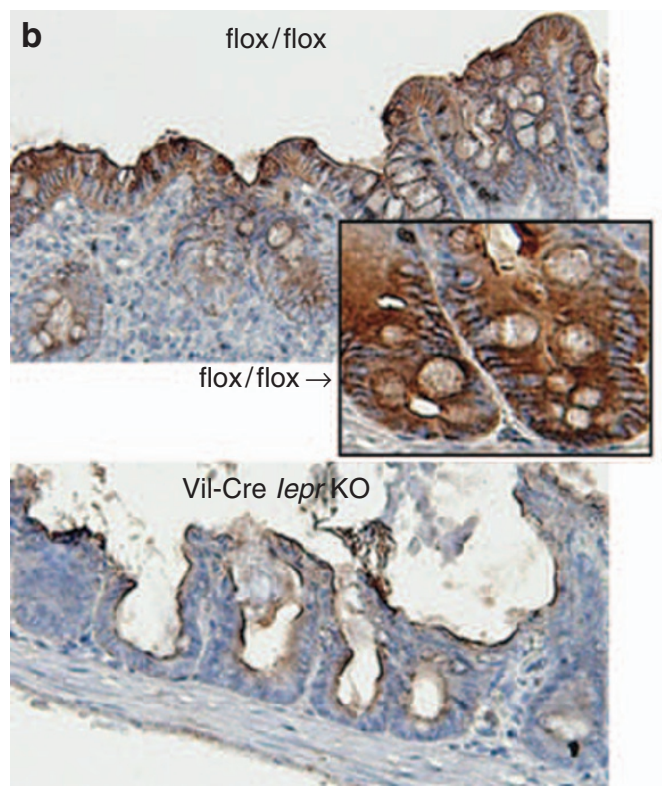

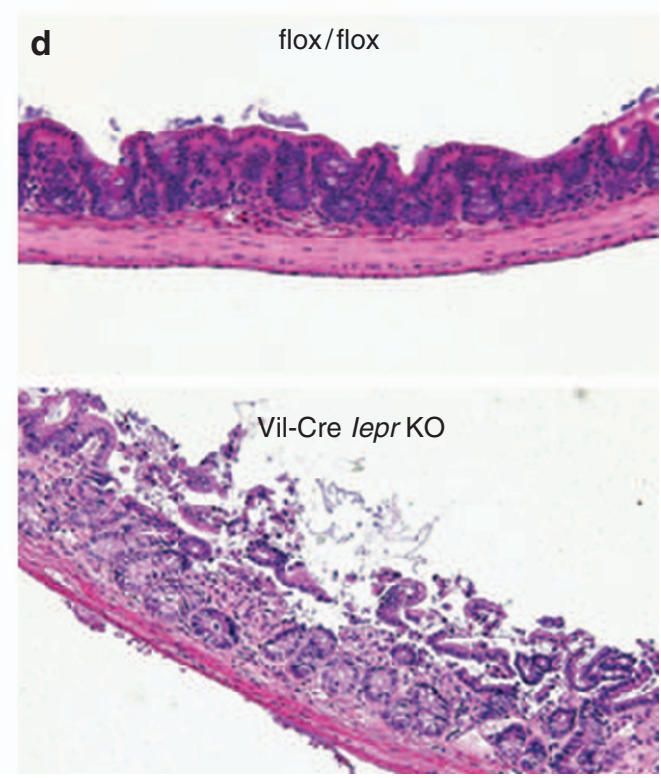

Figure 5 Intestine-specific deletion of leptin receptor rendered mice susceptible to amebiasis. Mice engineered with loxP sites flanking the first coding exon of the leptin receptor were mated with mice expressing the Vil-Cre recombinase. (a) Intestine-specific deletion of exon 1 was confirmed by PCR using primers flanking the LoxP sites $(\downarrow)$. The primers amplified a single band from the lepr $\Delta$ allele, whereas no product was generated from the control wild-type (WT) or floxed lepr allele. Genomic DNA was extracted from the cecum (Cec), liver (Liv), or the adipose tissue (Adi) associated with the intestine to examine the intestine-specific deletion of lepr. (b) Deletion of leptin receptor was further confirmed by its absence as demonstrated by immunohistochemistry in the Vil-Cre KO (bottom), but not the flox/flox control (top) cecum. Abundant basolateral staining of leptin receptor was seen in the flox/flox cecum (insert). Original magnifications: $\times 200$; insert: $\times 400$. (c) Vil-Cre KO mice and the Non-cre flox/flox controls were intracecally challenged with E. histolytica and killed 4 days after challenge. Intestinal infection was determined by culture positivity and cecal amebic antigen.

(d) Representative cecal histopathology demonstrated substantial mucosal erosion and epithelial loss in the infected Vil-Cre KO mice but none of the flox/flox controls. Original magnifications $\times 100$.

The role of leptin in promoting host resistance to infection has been demonstrated in several studies using leptin- and leptin receptor-deficient mice. ${ }^{38-40}$ Previous studies implicated the proinflammatory effect of leptin on cell-mediated immunity as being important for control of infection. However, leptin is a pleiotropic hormone that regulates the neuroendocrine axis centrally and immune/epithelial homeostasis peripherally. $3,7,8$ Although the proinflammatory cellular response may contribute to the clearance of the infection in some circumstances, the effects of leptin on prevention of epithelial apoptosis and promoting tissue repair might be required for mucosal defense against pathogens like E. histolytica. In our study, leptin appeared to be an essential factor for host resistance to intestinal amebiasis, as supported by the enhanced susceptibility in ob/ob and $\mathrm{db} / \mathrm{db}$ mice. Compared with the highly resistant wild-type B6 controls, an increase in mortality rate was observed in $\mathrm{db} / \mathrm{db}$ and $\mathrm{ob} / \mathrm{ob}$ mice after E. histolytica infection, and the mice exhibited profound mucosal destruction in the intestine. Interestingly, although no statistical significance was reached, the receptor deficient $\mathrm{db} / \mathrm{db}$ mice appeared to be more susceptible to both infection and mortality compared with the leptin-deficient $\mathrm{ob} / \mathrm{ob}$ mice. This finding suggested that the leptin receptor may signal to some extent in the absence of leptin, or the existence of additional ligands other than leptin that might partially compensate the leptin deficiency in ob/ob mice. A similar pattern has been observed for ciliary neurotropic factor (CNTF), where mice lacking the CNTF receptor, rather than mice lacking CNTF, exhibited profound motor neuron deficits at birth. ${ }^{4}$ Our data, together with the previous observations that $\mathrm{ob} / \mathrm{ob}$ mice have enhanced sensitivity to endotoxin-induced lethality ${ }^{42}$ and organ failure, ${ }^{43,44}$ suggest that leptin may be involved in the acute-phase response to systemic inflammation and mucosal injury.

The connection between leptin deficiency and disease susceptibility is likely to be complex. The functional leptin receptor (LRb or OB-Rb) is expressed on many immune cell types. Leptin has been shown, for instance, to modulate T-cell proliferation, promote Th1 responses, and protect a variety of cell types from 

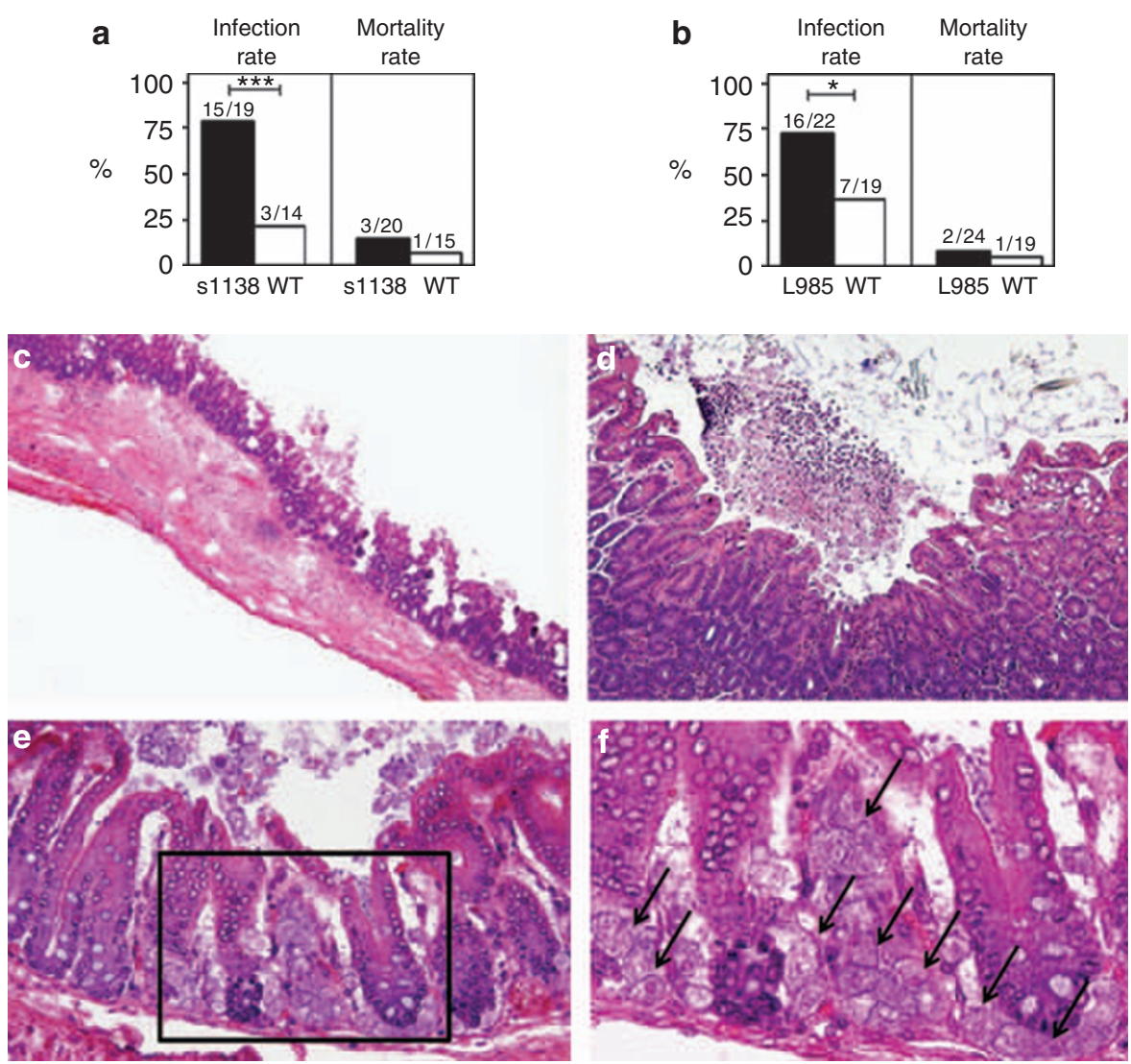

Figure 6 Signaling pathways through tyrosine 1138 and tyrosine 985 were both important in leptin-mediated protection. Mice with tyrosine residue substitutions, (a) S1138 or (b) L985, on the leptin receptor were more susceptible to intestinal infection with Entamoeba histolytica compared with wildtype (WT) littermates ( $P=0.001$ and 0.02 , respectively). Infection rate was determined by both cecal amebic antigen and cultural positivity. None of the variants had the rapid death phenotype as seen in the $\mathrm{db} / \mathrm{db}$ mice. ${ }^{*} P<0.05$; ${ }^{* \star *} P<0.001$ compared with wild-type littermates. (c) Most of the infected S1138 mice showed tissue destruction including mucosal erosion, ulceration, and tissue necrosis. (d) Infected L985 mice were mainly manifested with mucosal hyperplasia with very little mucosal destruction. (e, f) Loss of epithelial integrity was seen in some S1138 mice with aggressively invading trophozoites (arrows) along the lamina propria and basement membrane. Original magnifications: (c) $\times 40,(\mathbf{d}) \times 100,(\mathbf{e}) \times 200,(\mathbf{f}) \times 400$.

apoptosis. $3,8,11-13,45$ These observations led to the speculation that lack of effects of leptin on immune cells might account for the impaired resistance to amebic infection observed in ob/ob and $\mathrm{db} / \mathrm{db}$ mice. In order to determine the relative contribution of leptin effects on the immune vs. nonimmune system, we examined the susceptibility of bone marrow chimeras between $\mathrm{db} / \mathrm{db}$ and wild-type B6 mice. Both the infection and mortality rates were similar in $\mathrm{db} / \mathrm{db}$ mice reconstituted with either $\mathrm{db} / \mathrm{db}$ or wild-type bone marrow, indicating that leptin actions on radiosensitive hematopoietic cells are not sufficient to confer protection. Accordingly, LRb expressed on radioresistant cells, such as neurons or peripheral nonimmune cells, may have an essential role in protection.

LRb-expressing neurons in the brain have the potential to regulate susceptibility to infection directly or indirectly. With regard to the former, leptin could act centrally by regulating the activity of nerves projecting from the central nervous system to immune organs or the intestine for example. Alternatively, leptin's actions could be an indirect secondary effect of the multiple neuroendocrine and metabolic abnormalities that result from lack of central LRb function. For example, lack of central leptin function in ob/ob and $\mathrm{db} / \mathrm{db}$ mice increases activation of the hypothalamic-pituitary-adrenal axis, leading to increased glucocorticoid and decreased thyroid and growth hormone levels that could indirectly affect immune and intestinal functions important in amebic infection. ${ }^{46}$ To address the contributions of central leptin signaling to leptin-mediated protection, Tam-Cre mice with an intact leptin receptor present in the brain but disrupted in periphery were generated and tested for their susceptibility to amebiasis. An increase in both susceptibility and disease severity were observed in Tam-Cre mice compared with Non-cre littermates, indicating that intact central leptin signaling alone is not sufficient to protect the host from mucosal infection, further pointing to the importance of intact LRb signaling in the periphery for protection. $\mathrm{LRb}$ expression has been detected in the gut epithelium ${ }^{25,47}$ together with the fact that mucosal destruction in $\mathrm{db} / \mathrm{db}$ mice happens within $24 \mathrm{~h}$, suggesting that leptin might be acting locally on intestinal epithelial cells to mediate protection. This hypothesis was finally verified by infecting mice harboring a targeted deletion of lepr in intestinal epithelial cells (Vil-Cre KO), and showing that they exhibited significantly higher susceptibility and a greater level of mucosal destruction compared with their littermate controls. Taken together, these data demonstrate that leptin-mediated protection from amebiasis is mediated primarily through a direct action on the epithelial cells of the intestinal mucosa. 
Some insight into the mechanism by which leptin mediates mucosal protection has been gained from our genetic studies that identified Q223R, a common genetic polymorphism in the leptin receptor gene, to affect the susceptibility to amebiasis (P. Duggal et al., unpublished data). Children with the $\mathrm{R}$ allele showed significantly higher susceptibility than subjects homozygous for the $\mathrm{Q}$ allele, and this association was further verified in transgenic mice showing that the $\mathrm{R}$ allele carriers were more susceptible to amebic infection along with a greater level of epithelial apoptosis and reduced Akt phosphorylation in the gut. The leptin receptor has antiapoptotic effects via its activation of the PI3K/Akt pathways, ${ }^{17,28,29}$ and E. histolytica is known to kill host cells by inducing caspase 3 activation. ${ }^{48,49}$ We therefore hypothesize that the ancestral leptin receptor with the favorable Q allele protects the intestinal epithelium from E. histolytica-induced apoptosis by activating the PI3K/Akt pathway.

Increased susceptibility to amebiasis was also observed in both S1138 and L985 variants. The S1138 variant has impaired LRb/STAT3 signaling and disrupted expression of a variety of STAT3-inducible genes; the L985 mutant on the other hand, has enhanced STAT3 activation because of the loss of inhibitory signaling. It has been shown that the hypothalamic signaling of leptin through STAT3 has a critical role in regulation of neuroendocrine functions, ${ }^{31,50}$ which explains the obesity and infertility phenotype of the S1138 mouse. However, how the LRb/STAT3 signaling might function in periphery remains unclear. Previous studies have shown that the interleukin 6 (IL-6)/gp130/STAT3 signaling axis is important in maintaining intestinal homeostasis as (i) mucosal inflammatory cells responding to IL-6/IL-6R trans-signaling contribute to the development of colitis in a STAT3-dependent pathway, ${ }^{51,52}$ and (ii) enterocyte-specific STAT3 activation promotes epithelial survival and proliferation. ${ }^{53,54}$ As an IL-6 receptor family member, leptin receptor is presumably acting on the intestinal mucosa in a similar pattern. The enhanced susceptibility of S1138 mice to intestinal amebiasis indicated the importance of LRb/STAT3 signaling in leptin-mediated mucosal defense, but the downstream events accounting for the protection remain unknown. Studies are underway to identify the candidate effectors that are inducible by LRb/STAT3 and involved in the local mucosal defense (i.e., antiapoptosis, proproliferation, and antimicrobial peptide production).

The blocked inhibitory signaling in the L985 variant has been reported to result in enhanced STAT3 activation and accordingly a "gain-of-function" leptin-hypersensitivity phenotype characterized by low body weight, low circulating leptin, and resistance to diet-induced obesity. ${ }^{32,33}$ It has been demonstrated that the strength and duration of STAT3 activity determines if it is anti or proapoptotic, and enhanced STAT3 signaling because of loss of SOCS3 could induce apoptosis via repressing the PI3K/Akt pathway and reducing B-cell lymphoma 2 and cyclin D1 expression. ${ }^{55}$ This finding could partly explain why we saw an increased susceptibility to amebiasis in the L985 mice with disrupted SOCS3 binding and enhanced STAT3 signaling. Another important pathway through LRb
Y985 is SHP2-GRB-MAPK/ERK, whose functional effect in vivo has not been well defined. The uncoupled SHP2 binding to the L985 mutant disrupts MAPK/ERK activation, which has been considered as another prosurvival signal (in addition to $\mathrm{PI} 3 \mathrm{~K} / \mathrm{Akt}$ ) and is implicated in the survival of various types of epithelial cells. ${ }^{56-58}$ Thus, the increased susceptibility in L985 mice may be via attenuated MAPK/ERK signaling because of the L985 mutation. Note that the cecal histopathology was different for the L985 and S1138 infected mice (Figure 6c-f), suggesting the divergent effects of leptin action in control of epithelial resistance. Taken together, it is possible that multiple leptin signaling pathways are involved in protection against intestinal amebiasis, and the mechanisms by which 223R LRb fails to protect might not be the same for the S1138 and L985 receptors. Further study is necessary to clarify the relationship between STAT3/SOCS3, MAPK/ERK, and PI3K/Akt pathways in leptin-mediated resistance to amebiasis.

In conclusion, the data presented here indicate that differential susceptibility to amebiasis can be explained at least in part by variable leptin receptor function; leptin-mediated resistance is via its actions on intestinal epithelium rather than hematopoietic cells or the brain, and receptor signaling through STAT3 or SHP2/ERK are both involved in leptin-mediated resistance to E. histolytica infection. By showing the association of the adipocytokine leptin with susceptibility to a mucosal parasitic infection, and by demonstrating its site of action and signaling pathways, our work provides insights into the mechanisms by which malnutrition compromises the immune system.

\section{METHODS}

Animals. Six-week-old male C57BL/6J wild-type (B6), leptin-deficient (B6.V-Lep ${ }^{\mathrm{ob}} / \mathrm{J}, \mathrm{ob} / \mathrm{ob}$ ), and functional leptin receptor-deficient (B6.Cg-m Lepr $\mathrm{db}^{\mathrm{db}} / \mathrm{J}, \mathrm{db} / \mathrm{db}$ ) mice were purchased from Jackson Laboratories (Bar Harbor, ME). Animals were maintained under specific pathogenfree conditions at the University of Virginia. All protocols were approved by the institutional animal care and use committee.

C57BL/6J mice carrying a replacement of the wild-type $\mathrm{LRb}^{\mathrm{Y} 1138}$ with a serine residue (S1138) were generated by Bates et al. ${ }^{50}$ as previously described. C57BL/6J mice carrying a LRb ${ }^{985}$ Tyr $\rightarrow$ Leu substitution (L985) that replaces the wild-type Tyr985 were generated by Björnholm et al. ${ }^{59}$ as previously described. Interbreeding of heterozygous animals yielded wild-type, heterozygous, and homozygous mice for this study.

The Tam-Cre mouse model with an intact LRb in the brain but a disrupted $\mathrm{LRb}$ in periphery was generated using a Tam-inducible Cre-LoxP system. ${ }^{36}$ Briefly, the Lepr flox/flox mice with LRb exon 17 flanked by two loxP sequences ${ }^{35}$ were crossed to ROSA26Cre-ERT2/+ transgenic mice, in which a Cre-ER ${ }^{\mathrm{T} 2}$ fusion gene was introduced into ROSA26 locus. The Cre recombinase ubiquitinously expressed in ROSA26 Cre-ERT2/+ mice remains inactive until it is bound to Tam. ROSA26 Cre-ERT2/+ transgenic mice were backcrossed to Lepr flox/flox mice to produce Lepr flox/flox ROSA26Cre-ERT2/+ (Cre mice) and Leprflox/flox (Non-cre mice), segregating in the FVB/NJ background. Target deletion of exon 17 in peripheral tissues was induced by intraperitoneal administration of Tam ( $1 \mathrm{mg}$ per day for 5 consecutive days) to 6-8 weeks old Cre mice (designated as Tam-Cre mice). As exon 17 encodes the Jak2 binding site that is indispensable for the downstream LRb signal transduction, deletion of exon 17 results in a disrupted/dysfunctional LRb. ${ }^{35}$ Because of the poor permeability of the blood-brain barrier to Tam and its metabolites, Cre-ER ${ }^{\mathrm{T} 2}$ fusion protein in the brain cells remained inactive and thus the brain Lepr remained intact in Tam-treated Cre-Tam mice. ${ }^{36}$ 
Tam-treated Non-cre littermates and Cre mice with no Tam treatment were used as controls.

Intestine-specific deletion of Lepr was generated by two successive crosses between Vil-Cre transgenic mice (B6 background, The Jackson Laboratories) and Leprflox/flox mice (kindly provided by J. Friendman). Note that the Lepr flox/flox mice used here had the first coding exon of Lepr flanked by two loxP sites. ${ }^{37}$ As the first coding exon contains a signal sequence that is critical for the expression of all leptin receptor splicing variants, ${ }^{37}$ deletion of this exon by Vil-Cre-mediated recombination results in a null allele of leptin receptor in the intestine.

Animal model for intestinal amebic infection. The parasites used for the infection model and the method for intracecal inoculation have been described previously. ${ }^{60,23}$ After infection by E. histolytica, mice were monitored closely for general well-being; those who were judged to be in a moribund state (seemingly sick, severely dehydrated, and complete loss of activity) were euthanized before the scheduled termination time, whereas the rest were killed at $48 \mathrm{~h}$ (for B6 wild-type vs. db/db and ob/ob) or 3 to 4 days (for S1138, L985, Tam-Cre, and Vil-Cre mice) post-challenge.

Upon killing, each cecum was longitudinally bisected. Half was placed in Hollande's fixative (Sigma-Aldrich, St Louis, MO), paraffin embed$\mathrm{ded}$, and processed for hematoxylin and eosin staining. The other half of cecum was rinsed with $500 \mu \mathrm{l}$ phosphate-buffered saline; $200 \mu \mathrm{l}$ of cecal rinse was assayed for amebic antigen using the E. histolytica II ELISA kit (Techlab, Blacksburg, VA) and $300 \mu$ cultured in complete TYI-S-33 medium with supplemental antibiotics for 5 days at $37^{\circ} \mathrm{C}$.

Bone marrow chimera. Male C57BL/6 wild-type (Thy1.2), C57BL/6 (Thy1.1), and $\mathrm{db} / \mathrm{db}$ (Thy1.2) mice were used for the bone marrow transplantation. Recipient mice received total body irradiation (1,300 rad from a ${ }^{137} \mathrm{Cs}$ source) and were reconstituted intravenous within $4 \mathrm{~h}$ with $10^{7}$ bone marrow cells prepared from donor femurs and tibias, and then allowed to engraft for 8 weeks. During weeks $0-3$ of engraftment, chimeric mice were given trimethoprim/sulfamethoxazole-supplemented water for prophylaxis from opportunistic infection. Four groups were analyzed in this study: $\mathrm{db} / \mathrm{db}$ mice reconstituted with Thy1.1 B6 bone marrow cells; Thy1.1 B6 mice reconstituted with $\mathrm{db} / \mathrm{db}$ bone marrow cells; wild-type B6 mice reconstituted with congenic Thy1.1 mouse bone marrow cells; and $\mathrm{db} / \mathrm{db}$ mice reconstituted with $\mathrm{db} / \mathrm{db}$ bone marrow cells. Engraftment of hematopoietic cells from donor origin was confirmed on peripheral blood leukocytes at 6 weeks post-reconstitution, and on splenocytes and mesenteric lymph node cells upon killing, by fluorescence-activated cell sorting using conjugated Abs to Thy1.1 and Thy1.2 (BD Biosciences, San Diego, CA).

Immunohistochemical staining. Immunohistochemical staining for leptin receptor was performed with an $\mathrm{ABC}$ kit (Vector Laboratories, Burlingame, CA) per the manufacturer's instruction. Briefly, paraffin-embedded cecal sections were dewaxed with xylene and hydrated through alcohol gradient. Endogenous peroxidases were inactivated by incubating the slides in $0.3 \%$ hydrogen peroxide in methanol. After antigen retrieval with the Na-Citrate buffer $(10 \mathrm{mM}, \mathrm{pH}$ 6.5), the sections were blocked with $5 \%$ normal rabbit serum, then incubated with goat-anti-mouse leptin receptor antibody (R\&D, Minneapolis, MN) for $1 \mathrm{~h}$ at room temperature. Slides were then incubated with the biotinylated rabbit-anti-goat IgG for $30 \mathrm{~min}$, followed by $30 \mathrm{~min}$ of incubation with $\mathrm{ABC}$ reagent. Freshly made $3,3^{\prime}$-diaminobenzidine substrate solution was used to develop the sections and counterstaining was performed with hematoxylin (Vector Labs). After dehydration through alcohol gradient and clearing with xylene, slides were mounted with Cytoseal XYL (Richard-Allan Scientific, Kalamazoo, MI).

Statistics. All results are representative of at least two independent experiments with similar results. Data were analyzed with GraphPad Prism version 5.00 for Windows (GraphPad Software, San Diego, CA). Mann-Whitney test was performed to compare the means of two groups and Kruskal-Wallis test for comparison of three or more groups. Log-rank test was used to compare the difference in survival. A $P$-value of $<0.05$ was considered significant.

\section{ACKNOWLEDGMENTS}

We thank Ellyn Moore, Suzanne Stroup, Gina Wimer, and Wenhao Xu for their technical assistance. We also thank Alan Sher, Tom Braciale, Loren Erickson, Joanna Goldberg, Ulrike Lorenz, and Judith Woodfolk for scientific discussions and valuable advices. This work was supported by funding from the NIH grant Al026649 (to W.A.P.), R01 Al071373 (to E.R.H.), MARCE 2U54 Al057168-07 (to M.R. \& W.A.P.), R01 DK056731 (to M.G.M.), and 5P30, DK26687-29 New York Obesity Research Center, Molecular Biology/Molecular Genetics Core (to S.C.C.).

\section{DISCLOSURE}

The authors declared no conflict of interest.

(C) 2011 Society for Mucosal Immunology

\section{REFERENCES}

1. Chandra, R.K. Nutrition, immunity and infection: from basic knowledge of dietary manipulation of immune responses to practical application of ameliorating suffering and improving survival. Proc. Natl. Acad. Sci. USA 93, 14304-14307 (1996).

2. Chan, J. et al. Effects of protein calorie malnutrition on tuberculosis in mice. Proc. Natl. Acad. Sci. USA 93, 14857-14861 (1996).

3. Lord, G.M. et al. Leptin modulates the T-cell immune response and reverses starvation-induced immunosuppression. Nature 394, 897-901 (1998).

4. Mondal, D., Petri, W.A. Jr, Sack, R.B., Kirkpatrick, B.D. \& Haque, R. Entamoeba histolytica-associated diarrheal illness is negatively associated with the growth of preschool children: evidence from a prospective study. Trans. R. Soc. Trop. Med. Hyg. 100, 1032-1038 (2006)

5. Mondal, D., Haque, R., Sack, R.B., Kirkpatrick, B.D. \& Petri, W.A. Jr Attribution of malnutrition to cause-specific diarrheal illness: evidence from a prospective study of preschool children in Mirpur, Dhaka, Bangladesh. Am. J. Trop. Med. Hyg. 80, 824-826 (2009).

6. Moore, S.E. et al. Leptin, malnutrition, and immune response in rural Gambian children. Arch. Dis. Child 87, 192-197 (2002).

7. Tilg, H. \& Moschen, A.R. Adipocytokines: mediators linking adipose tissue, inflammation and immunity. Nat. Rev. Immunol. 6, 772-783 (2006).

8. Matarese, G., Moschos, S. \& Mantzoros, C.S. Leptin in immunology J. Immunol. 174, 3137-3142 (2005).

9. Myers, M.G., Cowley, M.A. \& Munzberg, H. Mechanisms of leptin action and leptin resistance. Annu. Rev. Physiol. 70, 537-556 (2008).

10. Chehab, F.F., Lim, M.E. \& Lu, R. Correction of the sterility defect in homozygous obese female mice by treatment with the human recombinant leptin. Nat. Genet. 12, 318-320 (1996).

11. Howard, J.K. et al. Leptin protects mice from starvation-induced lymphoid atrophy and increases thymic cellularity in ob/ob mice. J. Clin. Invest. 104, 1051-1059 (1999)

12. Papathanassoglou, E. et al. Leptin receptor expression and signaling in lymphocytes: kinetics during lymphocyte activation, role in lymphocyte survival, and response to high fat diet in mice. J. Immunol. 176, 7745-7752 (2006).

13. Fernandez-Riejos, P., Goberna, R. \& Sanchez-Margalet, V. Leptin promotes cell survival and activates Jurkat T lymphocytes by stimulation of mitogenactivated protein kinase. Clin. Exp. Immunol. 151, 505-518 (2008).

14. Martin-Romero, C., Santos-Alvarez, J., Goberna, R. \& Sanchez-Margalet, V. Human leptin enhances activation and proliferation of human circulating T lymphocytes. Cell Immunol. 199, 15-24 (2000).

15. Lam, Q.L., Liu, S., Cao, X. \& Lu, L. Involvement of leptin signaling in the survival and maturation of bone marrow-derived dendritic cells. Eur. J. Immunol. 36, 3118-3130 (2006).

16. Conus, S., Bruno, A. \& Simon, H.U. Leptin is an eosinophil survival factor. J. Allergy Clin. Immunol. 116, 1228-1234 (2005).

17. Bruno, A., Conus, S., Schmid, I. \& Simon, H.U. Apoptotic pathways are inhibited by leptin receptor activation in neutrophils. J. Immunol. 174, 8090-8096 (2005)

18. Najib, S. \& Sanchez-Margalet, V. Human leptin promotes survival of human circulating blood monocytes prone to apoptosis by activation of p42/44 MAPK pathway. Cell Immunol. 220, 143-149 (2002).

19. Lo, C.K. et al. Leptin signaling protects NK cells from apoptosis during development in mouse bone marrow. Cell Mol. Immunol. 6, 353-360 (2009). 
20. Otero, M. et al. Towards a pro-inflammatory and immunomodulatory emerging role of leptin. Rheumatology (Oxford) 45, 944-950 (2006).

21. De Rosa, V. et al. A key role of leptin in the control of regulatory T cell proliferation. Immunity 26, 241-255 (2007).

22. Haque, R. et al. Correlation of interferon-gamma production by peripheral blood mononuclear cells with childhood malnutrition and susceptibility to amebiasis. Am. J. Trop. Med. Hyg. 76, 340-344 (2007).

23. Guo, X., Stroup, S.E. \& Houpt, E.R. Persistence of Entamoeba histolytica infection in CBA mice owes to intestinal IL-4 production and inhibition of protective IFN-gamma. Mucosal. Immunol. 1, 139-146 (2008).

24. Guo, X. et al. Protection against intestinal amebiasis by a recombinant vaccine is transferable by $T$ cells and mediated by gamma interferon. Infect. Immun. 77, 3909-3918 (2009).

25. lqbal, J. et al. An intrinsic gut leptin-melanocortin pathway modulates intestinal microsomal triglyceride transfer protein and lipid absorption. J. Lipid Res. 51, 1929-1942 (2010).

26. Brun, P. et al. Increased intestinal permeability in obese mice: new evidence in the pathogenesis of nonalcoholic steatohepatitis. Am. J. Physiol. Gastrointest. Liver Physiol. 292, G518-G525 (2007).

27. El, H.M. et al. Leptin modulates the expression of secreted and membrane-associated mucins in colonic epithelial cells by targeting PKC, PI3K, and MAPK pathways. Am. J. Physiol. Gastrointest. Liver Physiol. 293, G365-G373 (2007).

28. Ogunwobi, O.O. \& Beales, I.L. The anti-apoptotic and growth stimulatory actions of leptin in human colon cancer cells involves activation of JNK mitogen activated protein kinase, JAK2 and PI3 kinase/Akt. Int. J. Colorectal Dis. 22, 401-409 (2007).

29. Sukhotnik, l. et al. Effect of leptin on intestinal re-growth following massive small bowel resection in rat. Pediatr. Surg. Int. 22, 9-15 (2006).

30. Baumann, H. et al. The full-length leptin receptor has signaling capabilities of interleukin 6-type cytokine receptors. Proc. Natl. Acad. Sci. USA 93, 8374-8378 (1996).

31. Myers, M.G. Jr Leptin receptor signaling and the regulation of mammalian physiology. Recent Prog. Horm. Res. 59, 287-304 (2004).

32. Banks, A.S., Davis, S.M., Bates, S.H. \& Myers, M.G. Jr Activation of downstream signals by the long form of the leptin receptor. J. Biol. Chem. 275, 14563-14572 (2000).

33. Bjorbak, C. et al. SOCS3 mediates feedback inhibition of the leptin receptor via Tyr985. J. Biol. Chem. 275, 40649-40657 (2000).

34. Bijian, K., Takano, T., Papillon, J., Khadir, A. \& Cybulsky, A.V. Extracellular matrix regulates glomerular epithelial cell survival and proliferation. Am. J. Physiol. Renal Physiol. 286, F255-F266 (2004).

35. McMinn, J.E. et al. An allelic series for the leptin receptor gene generated by CRE and FLP recombinase. Mamm. Genome. 15, 677-685 (2004).

36. Guo, K. et al. Disruption of peripheral leptin signaling in mice results in hyperleptinemia without associated metabolic abnormalities. Endocrinology 148, 3987-3997 (2007).

37. Cohen, P. et al. Selective deletion of leptin receptor in neurons leads to obesity. J. Clin. Invest 108, 1113-1121 (2001).

38. Mancuso, P. et al. Leptin-deficient mice exhibit impaired host defense in Gram-negative pneumonia. J. Immunol. 168, 4018-4024 (2002).

39. Moore, S.I., Huffnagle, G.B., Chen, G.H., White, E.S. \& Mancuso, P. Leptin modulates neutrophil phagocytosis of Klebsiella pneumoniae. Infect. Immun. 71, 4182-4185 (2003).

40. Ikejima, S. et al. Impairment of host resistance to Listeria monocytogenes infection in liver of db/db and ob/ob mice. Diabetes 54, 182-189 (2005).

41. DeChiara, T.M. et al. Mice lacking the CNTF receptor, unlike mice lacking CNTF, exhibit profound motor neuron deficits at birth. Cell 83, 313-322 (1995).
42. Faggioni, R. et al. Leptin deficiency enhances sensitivity to endotoxininduced lethality. Am. J. Physiol. 276, R136-R142 (1999).

43. Yang, S.Q., Lin, H.Z., Lane, M.D., Clemens, M. \& Diehl, A.M. Obesity increases sensitivity to endotoxin liver injury: implications for the pathogenesis of steatohepatitis. Proc. Natl. Acad. Sci. USA 94, 2557-2562 (1997).

44. Wang, W. et al. Role of leptin deficiency in early acute renal failure during endotoxemia in ob/ob mice. J. Am. Soc. Nephrol. 15, 645-649 (2004).

45. Sanchez-Margalet, V. et al. Role of leptin as an immunomodulator of blood mononuclear cells: mechanisms of action. Clin. Exp. Immunol. 133, $11-19$ (2003).

46. Flier, J.S. Lowered leptin slims immune response. Nat. Med. 4, 1124-1125 (1998).

47. Hansen, G.H., Niels-Christiansen, L.L. \& Danielsen, E.M. Leptin and the obesity receptor (OB-R) in the small intestine and colon: a colocalization study. J. Histochem. Cytochem. 56, 677-685 (2008).

48. Huston, C.D., Houpt, E.R., Mann, B.J., Hahn, C.S. \& Petri, W.A. Jr Caspase 3-dependent killing of host cells by the parasite Entamoeba histolytica. Cell Microbiol. 2, 617-625 (2000).

49. Boettner, D.R. \& Petri, W.A. Entamoeba histolytica activates host cell caspases during contact-dependent cell killing. Curr. Top. Microbiol. Immunol. 289, 175-184 (2005).

50. Bates, S.H. et al. STAT3 signalling is required for leptin regulation of energy balance but not reproduction. Nature 421, 856-859 (2003).

51. Matsumoto, S. et al. Essential roles of IL-6 trans-signaling in colonic epithelial cells, induced by the IL-6/soluble-IL-6 receptor derived from lamina propria macrophages, on the development of colitis-associated premalignant cancer in a murine model. J. Immunol. 184, 1543-1551 (2010).

52. Atreya, R. et al. Blockade of interleukin 6 trans signaling suppresses T-cell resistance against apoptosis in chronic intestinal inflammation: evidence in Crohn disease and experimental colitis in vivo. Nat. Med. 6, 583-588 (2000).

53. Bollrath, J. et al. gp130-mediated Stat3 activation in enterocytes regulates cell survival and cell-cycle progression during colitis-associated tumorigenesis. Cancer Cell 15, 91-102 (2009).

54. Grivennikov, S. et al. IL-6 and Stat3 are required for survival of intestinal epithelial cells and development of colitis-associated cancer. Cancer Cell 15, 103-113 (2009).

55. Lu, Y. et al. Loss of SOCS3 gene expression converts STAT3 function from anti-apoptotic to pro-apoptotic. J. Biol. Chem. 281, 36683-36690 (2006).

56. di Mari, J.F., Davis, R. \& Safirstein, R.L. MAPK activation determines renal epithelial cell survival during oxidative injury. Am. J. Physiol. 277, F195-F203 (1999).

57. Edelblum, K.L. et al. Raf protects against colitis by promoting mouse colon epithelial cell survival through NF-kappaB. Gastroenterology 135, 539-551 (2008).

58. Howie, H.L., Shiflett, S.L. \& So, M. Extracellular signal-regulated kinase activation by Neisseria gonorrhoeae downregulates epithelial cell proapoptotic proteins Bad and Bim. Infect. Immun. 76, 2715-2721 (2008).

59. Björnholm, M. et al. Mice lacking inhibitory leptin receptor signals are lean with normal endocrine function. J. Clin. Invest. 117, 1354-1360 (2007).

60. Houpt, E.R. et al. The mouse model of amebic colitis reveals mouse strain susceptibility to infection and exacerbation of disease by CD4+ T cells. J. Immunol. 169, 4496-4503 (2002). 